ne rend pas compte non plus de l'ensemble des caractères que l'on observe dans les cailloutis pléistocènes dits fluvio-glaciaires.

Le glacier de Valsorey nous fournit par contre la succession des phases par lesquelles le fluvio-glaciaire a pris naissance. A partir des moraines intraglaciaires probablement issues elles-mêmes de la moraine de fond se forment les moraines superficielles portées par des socles de glace; à l'aval de celles-ci et à la surface du glacier mort, le torrent et ses affluents remanient les cailloutis morainiques, c'est à ces derniers qu'il convient de réserver le nom de fluvio-glaciaire. Enfin le Valsorey nous montre l'étroite connexion entre le fluvio-glaciaire proprement dit et les dépôts varvés.

Il va de soi que dans le domaine du Pléistocène, il ne sera pas toujours facile de faire pratiquement la part de ce qui revient au fluvio-glaciaire plutôt qu'aux moraines intraglaciaires. Cette difficulté ne saurait cependant autoriser le maintien de la grave confusion entraînée par la notion telle qu'elle est actuellement admise.

\title{
BIBLIOGRAPHIE SOMMAIRE
}

1909 - Penck, Albrecht et Brückner, Eduard: Die Alpen im Eiszeitalter. Leipzig. - 1935 GaGNEBIN, EliE: Ossements de Mammouth trouvés dans la moraine de Renens et recensement des restes de Mammouth connus dans la région lémanique. Laboratoire de Géologie de l'Université de Lausanne. Bull. No 54. - 1946 - JAYET, AdRiEn : Les dépôts quaternaires et la théorie des emboitements. Geographica Helvetica. I, No 4. - 1946 - Les stades de retrait würmiens aux environs de Genève. Ecl. Geol. Helvetiae. Vol. 39, No 2. - 1947 - Une nouvelle conception des glaciations quaternaires. Ecl. Geol. Helvetiae. Vol. 40, No 2. - 1952 - Quelques caractéristiques peu connues des dépôts glaciaires pléistocènes et actuels. Ecl. Geol. Helvetiae. Vol. 45, No 2.

\section{DAS PROBLEM DES FLUVIO-GLAZIALS}

Die Schotter des Pleistocäns der schweizerischen Hochebene werden meistens als fluvio-glaziale Ablagerungen betrachtet. Sie haben sich wahrscheinlich unter der Wirkung von Bergbächen, unterhalb der abgelagerten Gletschermoränen gebildet.

Eingehende Beobachtungen in den Schotterablagerungen zeigen, daß diese Erklärung kaum die richtige sein kann. Sie erklärt weder die Abwesenheit der Flußfauna noch das Vorhandensein gletscherhafter Erscheinungen in allen Schichten, d. h. der je nach der Höhe, Länge des Niveaus verschieden mächtigen Schotter, die keine noch so starke Strömung bewirkt haben kann.

Andere Beobachtungen am Valsorey- und Vélangletscher zeigten, wie ein typisches Fluvioglazial sich bildet. Die im Innern des Gletschers befindlichen Moränen werden von den übereinanderliegenden Rutschungen der Gletscherbänke abwärts gezogen. Sie kommen allmählich zum Vorschein. Es entsteht dann ein dreieckiger Eissockel, der die neue Stirnmoräne trägt. Im Laufe der Jahre schmilzt dieser Sockel allmählich, aber der ganze talwärts liegende Teil verwandelt sich in einen toten Gletscher, an dessen Oberfläche der Hauptstrom und die Nebenflüsse die Schotter bearbeiten. Das Fluvioglazial hat sich also an der Oberfläche der Eisschicht gebildet. Endlich sind an dieser Oberfläche in kleinen Seitenmulden auch fein geschichtete Ablagerungen entstanden. Es ist demnach möglich, die Bildung der pleistozänen Schotter auf dieselbe Weise zu erklären. Man darf nur die an der Oberfläche bearbeiteten Schotter als zum Fluvioglazial gehörig betrachten. Die anderen Schotter sind entweder Innenmoränen oder Stirn- und Seitenmoränen.

\section{DER GANZHEITSBEGRIFF IN DER BIOCOENOLOGIE UND IN DER LANDSCHAFTSKUNDE}

\author{
EMIL Schmid
}

\section{DIE BEGRIFFE}

Als Ganzheiten werden Objekte bezeichnet, welche umgrenzt und nicht weiter teilbar sind, ohne daß ihre Eigenschaften und Funktionen sich verändern. Artefakte können Ganzheiten sein, Gebrauchsgegenstände, Instrumente, Kunstwerke, Kompositionen, Atome, Moleküle, Planeten, Lebewesen. Ein Planaria-, ein Hydra-Individuum sind Ganzheiten; wenn wir sie halbieren, sind sie zerstört, selbst wenn sich die Hälften zu zwei neuen Individuen regenerieren. 
Als Organismen bezeichnet man lebendige Ganzheiten. Sie werden charakterisiert durch ihre relativ dauerhafte Konstitution, durch ihre Autonomie und Eigengesetzlichkeit, durch ihre Regulations- und Reproduktionsfähigkeit, durch ihre Fähigkeit zur Energienahme und Assimilation, durch ihre Organisation der Arbeitsteilung, ihr bestimmtes und begrenztes Gefüge. Einen Organismus können wir pars pro toto erkennen. Während die nichtorganismischen, nichtautonomen, von der Umwelt völlig abhängigen abiotischen Ganzheiten von der Art der Atome und Moleküle von Kernkräften und elektromagnetischen Kräften zusammengehalten werden, kommen bei den Lebewesen der untersten Stufe, den Protobionten, noch dazu Hüllen, Zellwände, Organelle, und weiter bei den Metabionten ein Gefüge mit Arbeitsteilung nach Organen, bei den Sozietäten des Menschen organisierte Arbeitsteilung, Energiebeschaffung, Sprache, Rechtsordnung, Verkehrsmittel usw. Immer sind es Kräfte, Hüllen, Gefüge, zentrale Organisationen der Glieder, welche ein unteilbares Ganzes umgrenzen und zusammenhalten und dieses steht durch besondere Eigenschaften der Grenzflächen, Membranen usw. mit der Außenwelt in Kontakt. Immer ist auch eine Abstammungslinie vorhanden. Die Bedingtheit durch das Milieu ist viel geringer als bei den abiotischen Ganzheiten und bei den landschaftlichen Abschnitten der Erdoberfläche.

Die Biocoenose. In den Lebensgemeinschaften finden sich nach Taxonomie und Altersphasen verschiedenartige Organismen in vielzähligen Populationen zusammen, und zwar an den ihnen gemäßen Standorten. An ähnlichen Biotopen treffen wir auf ähnliche Garnituren von Arten und Wuchsformen, an speziellen Standorten auch auf ganz einzigartige Artenkombinationen. Diesen Lebensgemeinschaften fehlen Abgrenzungen und zusammenhaltende Kräfte, welche aus ihnen selbst kommen. Es fehlt ihnen eine Autonomie, eine Eigengesetzlichkeit. Sie können sich nicht als ein Ganzes reproduzieren, sie sind teilbar, eine Arbeitsteilung besteht nicht. Die Abgrenzungen werden von außen her, von der Umwelt gestellt. Die Biocoenose ist also weder ein Organismus, noch eine Ganzheit, sondern nur ein \pm einheitliches summatives Beieinanderleben autonomer Teilnehmer mit Gleichgewichtstendenz. Die Arten haben sich zusammengefunden auf Grund der Auswahl, welche das Milieu bewerkstelligt hat. Die Teilnehmer können durch andere mit ähnlichen Ansprüchen ersetzt werden, sie können zahlreich oder arm an $\mathrm{Zahl}$ sein. Die Biocoenose ist nicht stetig, sondern in ständiger Veränderung begriffen. Je nach der Herkunft der Arten, ihre Phylogenie, ihrer Genetik, ihrer Chorologie und Verbreitungsgeschichte, je nach den œkologischen Ansprüchen ihrer Arten, nach ihren Wuchsformen, nach ihrem Bioklima sind die Kombinationen der Teilnehmer zustande gekommen. Bei der Analyse müssen wir deshalb auf die einzelnen autonomen Teilnehmer zurückgehen und ihr Verhalten untersuchen, und zwar in der Vollständigkeit inbezug auf die einzelnen Charaktere, wie etwa Gesamtverbreitung, oekologische Ansprüche usw. Die von ihnen gebildete Artenkombination, die Lebensgemeinschaft oder Biocoenose konstatieren wir als einheitlich in ihrem Gleichgewicht der Mitglieder untereinander und in der Kombination nach außen. Die Biocoenose ist ein einzigartiges Phänomen und die Einzigartigkeit wächst mit der Zahl der Teilnehmer. Was eine ganzheitliche Struktur vortäuscht, sind die Einheitlichkeit, die spezifischen Korrelationen der Teilnehmer zum Biotop, sind ferner die Korrelationen zwischen einzelnen Teilnehmern und das von allen Teilnehmern erzeugte Bioklima. Dabei ist jedoch zu sagen, daß jeder Teilnehmer auch in andern Artenkombinationen mit entsprechendem Lebensraum vorkommen kann, daß er autonom bleibt und daß deshalb die Gemeinschaft als solche nicht autonom sein kann. Das Zusammentreffen von Organismen am Standort, deren Abhängigkeit von abiotischen und biotischen Standortsverhältnissen, ihre durch Konkurrenz und maximale Leistung erzeugte Struktur genügen nicht, um ein ganzheitliches Gebilde zu schaffen; auch wenn die Biocoenose sich resistent erweist gegen das Eindringen von andern Arten, auch wenn sie Zerstörungen in ihrem Bestand ausgleichen, auch wenn sie über 
größere Strecken hinweg sich ausbreiten kann, so ist sie doch immer eine summative Erscheinung, und alle diese Bewegungen gehen vor sich über die einzelnen Teilnehmer. Diese sind es, welche das Milieu verändern, welche die Strukturen bilden auf dem von ihnen zubereiteten Standort. Jede Aktivität der Lebensgemeinschaft ist aus der summativen Aktivität der autonomen Teilnehmer erklärbar, und wenn auch diese Lebensgemeinschaften mehr oder weniger ganz von ihrem Milieu abhängig sind, so bleiben ihre Teilnehmer doch autonome Gebilde.

Die Landschaft. Bei der Landschaft haben wir es mit ähnlichen Problemen zu tun wie bei der Biocoenose, welche ja ein Teil der Landschaft ist. Während bei der Biocoenose es sich um eine Kombination von heterotypischen Organismen handelt, haben wir es bei der Landschaft mit einer Zusammenstellung von Komponenten zu tun, welche nichts anderes sein kann als jeweils ein vom Menschen aus gesehener Ausschnitt aus der Erdoberfläche. In der Landschaft erkennen wir nur Komponenten und Kräfte, welche sich auf die ganze Erdoberfläche beziehen und somit kosmischer Art sind. Licht, Temperatur, Feuchtigkeit, Erosion, Sedimentation, tektonische Verhältnisse usw. sind in Korrelation mit Drehung und Umlauf unseres Planeten; aber auch die organischen Siedler auf dieser Oberfläche, Pflanzen und Tiere, können nur verstanden werden im Zusammenhang mit der ganzen Erdoberfläche. Ihre phylogenetische Evolution, ihre Verbreitung, ihre Produktionsleistung sind Funktionen der ganzen Umwelt. Weniger noch als die Biocoenosen sind diese Ausschnitte der Erdoberfläche stetige Gebilde. Sie verändern sich fortdauernd und Quantität und Qualität dieser Veränderungen werden vom Ganzen, vom Sonnensystem und von der Erde her, gesteuert. Während nun die Komponenten einer Landschaft in ihrer Vollständigkeit einen gewissen Ganzheitscharakter aufweisen, kann dies nicht behauptet werden von ihrem Zusammentreten als Landschaft. Jede Komponente kann auch in anderen Ausschnitten figurieren, so daß die Landschaft ohne einheitliche Begrenzung, ja ohne theoretische Grenzen dasteht als ein Objekt, von dem beliebige Stücke weggenommen werden können, d. h. das größer oder kleiner gewählt werden kann, je nachdem, was ich mit ihm vorhabe. Jeder Teil setzt sich ja fort und wird vollständig erst im Bereiche der ganzen Erdoberfläche. Wir gehen deshalb bei der Landschaftskunde zurück auf die Komponenten, so wie in der Biocoenologie auf die einzelnen Teilnehmer, und konstatieren in den Landschaften die Zusammenstellungen der Komponenten. Die Landschaft ist kein Individuum, keine Ganzheit und erst recht kein Organismus. Die Umgrenzung ist eine anthropozentrische Angelegenheit. Sie wird frei ausgewählt je nach dem $Z$ weck der Bearbeitung eines Ausschnittes der Erdoberfläche. Wenn typisiert wird, so typisiert man die Komponenten. Man spricht z. B. von der Juraformation, von einer Plateaulandschaft, von den Erosionsformen eines jugendlichen Gebirges. Die monographische Bearbeitung kann nur von der Analyse der Komponenten ausgehen. Erst dann, wenn astronomische, geophysische, geotektonische, stratigraphische, geomorphologische, bodenkundliche, hydrologische, klimatologische, biocoenologische und andere Verhältnisse bearbeitet sind, kann eine Gebietsmonographie synthetisiert werden. Nur für praktische $Z$ wecke lohnt es sich, einzelne dieser Gesichtspunkte herauszugreifen. Bei den Einzelanalysen der Komponenten einer Landschaft zeigt es sich aufs deutlichste, wie eng die Beziehungen zur ganzen Erdoberfläche sind und wie sehr die Berücksichtigung der vollständigen Komponenten die $\mathrm{Zu}$ sammenhänge dem Verständnis näherbringt. Jedes einzelne Objekt hat seine bestimmte Lage, und diese ist von dokumentarischer Bedeutung. Ein Gebirge in seiner Tektonik wird nur verständlich, wenn wir die Tektonik des Erdballes überblicken. Wie das Lokalklima eines Ausschnittes allein vom Klima der Erdoberfläche her verstanden wird, so zeigen auch die Lebewesen in ihrer Entstehung, in ihren Wanderungen und in ihrer Ausbreitung vom größeren Milieu vorgezeichnete Wege. Ihre Gemeinschaften sind in Zusammensetzung und Physiognomie die besten Zeiger der 
Lebensbedingungen, und sie können nur im Zusammenhang mit ihrer biogeographischen Großgliederung und mit dem Klima und Boden verstanden werden.

Zusammenfassend läßt sich sagen, daß abiotische und biotische Ganzheiten durch besondere Kräfte, Hüllen, Gefüge, Organisationen zusammengehalten werden, während Landschaften und Biocoenosen solche nicht aufweisen und ihnen ganzheitlich machende Bindungen fehlen. Wir haben es zu tun mit Zusammenstellungen summativen Charakters und infolgedessen kann bei ihnen nicht von Individuen gesprochen werden. Es kann deshalb auch keine Klassen, keine Klassifikation und Klassenhierarchie, keine Generalisationen geben.

Die Oekumene. Eine ganz neue Situation schafft der Mensch sowohl für seine lebende wie auch für die anorganische Umwelt. Der natürliche Ausgleich zwischen den Organismen und zwischen den Organismen und ihrem Milieu, welcher noch in der Sammlerphase vorhanden war, ist immer mehr durch die viel brutaleren Einbrüche des Kulturmenschen ersetzt worden. Nicht nur die natürliche Vegetation, sondern die ganze Landschaft wurde verändert in einem solchen $\mathrm{Maße}$, daß sie als ein Gebilde betrachtet werden muß, das dem Menschen seine Gestaltung verdankt. Die Kulturlandschaft ist deshalb theoretisch und praktisch von der Naturlandschaft abzutrennen und im Zusammenhang mit dem Menschen zu behandeln durch den Soziologen, den Anthropologen, den Ethnographen zusammen mit dem sich auf die Kulturlandschaft spezialisierenden Geographen.

\section{DIE ANWENDUNG DER BEGRIFFE}

\section{IN DER BIOCOENOLOGIE UND IN DER LANDSCHAFTSKUNDE}

Die Fehlerquellen bei der Apperzeption der biocoenologischen und landschaftskundlichen Erscheinungen sind zahlreich. Je komplizierter (und mit wissenschaftlichen Mitteln unzugänglicher) ein Objekt wird, desto mehr beteiligt sich dabei eine gewisse Voreingenommenheit. Nur zu oft ist bei der Aufnahme durch die Sinnesorgane das Bedürfnis vorhanden, zu ergänzen, zu komplettieren, ähnlich wie eine zur Hälfte zerstörte Netzhaut das Objekt nicht halbiert, sondern ganz, wenn auch kleiner, sieht. Vermöge seiner Begabung, seiner Kenntnisse, seiner Erfahrung apperzipiert jeder anders. Dazu kommt noch, daß wir viel zu sehr geneigt sind, mit einer überkommenen Begriffswelt zu arbeiten, mit Kategorien, welche den Phänomen nicht adäquat sind. Wir apperzipieren als Ganzheiten, verleitet von falschen Analogien mit ganzheitlichen Gebilden, welche um uns sind. Dazu kommt noch, daß wir uns nur zu leicht die Vorstellung eines fiktiven Ganzen bilden, aus Teilen, welche wir objektiv konstatieren können, einen Allgemeinbegriff, der zu umfangreicher Benützung verführt und damit zu einer Vereinfachung der Phänomene, die eine Vergewaltigung der Natur bedeuten.

Weiterhin lassen wir uns verleiten, von der Einzigartigkeit unserer Objekte auf deren Ganzheit zu schließen. Dieses Abgleiten zu Ganzheitsvorstellungen erfolgt um so sicherer, je komplizierter das Objekt durch eine langdauernde Entwicklungsgeschichte ist. Ganz besonders gilt das, wenn ein Objekt durch die hohe Komplikation unikal geworden ist. Die suggestive Wirkung der Einzigartigkeit kann so weit gehen, daß wir ein Objekt seiner Einzigartigkeit halber als Ganzheit betrachten, z. B. einen Ausschnitt der Erdoberfläche von beliebiger Größe, sei er auch noch so klein, oder einen Ausschnitt aus der Vegetation der Erde. Wir vergessen dabei, daß diese Objekte keine aus ihrem Wesen entspringende, sondern nur eine durch abiotische Faktoren bewirkte Abgrenzung aufweisen, daß sie teilbar sind, und daß ihnen ganzmachende Charaktere fehlen. Einzigartig ist jeder Ausschnitt, aber er erfüllt damit den Begriff der Ganzheit nicht.

Es ist das einheitliche Objekt, von dem der Biocoenologe und der Landschaftskundler ausgehen. Die einheitliche Vegetation etwa eines Buchenwaldes, die sich über 
große Strecken hin manifestieren kann, die eintönige Wüstenlandschaft, sie sind es, welche der Forscher als sein Studienobjekt betrachtet, und darin liegt die Hauptschwierigkeit des behandelten Problemes. Wo einheitliche Vegetation in gleichmäßi. gem Milieu und gleicher Flora über große Strecken hin sich ausdehnt, wo große Landschaftskomponenten in einheitlicher Verbindung sich dem Auge aufdrängen, liegt die Versuchung zunächst, diese Phänomene für die Differenzierung zu benützen. Im übrigen sind inbezug auf die Artengarnitur einheitliche Vegetationen wohl in den extratropischen Gebieten häufig, aber in den Tropen erstreckt sich kontinuierlich artenwechselnde Vegetation übr weite Gebiete, von den Feuchtwäldern bis zu den Tropenwäldern, Waldsteppen, Steppen, Halbwüsten und Wüsten, und in den alten, konsolidierten Vegetationen Südostasiens bis weit in die subtropischen, temperierten Gebiete hinein. Aber eine synthetische Einheit im Sinne eines zusammengesetzten mannigfaltigen Objektes, dessen Komponenten in Beziehung zueinander stehen durch das Angepaßtsein aneinander, durch das labile Gleichgewicht kann einzigartig sein, braucht aber nicht ganzheitlich zu sein. Und im Falle der biocoenologischen und landschaftskundlichen Phänomene ist das auch nicht der Fall. Warum sind nun diese Objekte keine Ganzheit? Weil sie durch keine der für die Ganzheiten charakteristischen Kräfte, Hüllen, Organisationen zusammengehalten werden, weil sie nicht autonom sind, nicht unteilbar und nicht begrenzt. Das ist auch der Grund, warum wir keine Klassen bilden und keine Klassenhierarchie zu einem System aufbauen können. Ganz abgesehen von der Frage der Klassifikabilität ist es unmöglich, für die Vegetationsbeschreibungen allein auf die inbezug auf die Artengarnitur einheitlichen Ausschnitte abzustellen. Überall sehen wir Übergänge, und die Übergänge nehmen meist mehr Raum ein als die einheitlichen Partien. Die Grenzlinien sind vorwiegend vom Milieu gezogen.

In der Vegetationskunde hat das verwendete Klassifikationsprinzip, nämlich das floristisch-statistische, ja nur lokale Wirksamkeit soweit eben eine bestimmte Florenstruktur reicht. Das hat zur Folge, daß, wenn man die höheren Klassen bildet, ganz heterogene Artengarnituren zusammenkommen. Für diese bleibt zuletzt nur noch die Ähnlichkeit des abiotischen Milieus übrig, um das Gebilde zusammenzuhalten, so etwa bei den Thlaspeetalia der Braunschen Klassifikation. Ganz abgesehen davon kann man bei einem einheitlichen Objekt ohne Ganzheitscharakter nicht versuchen, dasselbe zu erfassen von einem einzigen Merkmal her wie dem floristisch-statistischen. Nur ganzheitliche Objekte lassen eine solche pars pro toto-Determinierung zu. Was wir erhalten ist ja auch nur ein bloßer Abklatsch der Natur durch eine floristische Beschreibung, begleitet von Angaben über das Milieu, aber ohne jede Möglichkeit zu einer Generalisierung. Fast immer fehlen auch Angaben über die vollständige Verbreitung der klassifizierten Einheiten. Das Einteilungsprinzip wechselt. Einmal ist es floristischstatistisch und dann wieder, bei den höhern Einheiten, müssen die Standorte herhalten. Inkonsequent ist es auch, wenn die Moosgesellschaften innerhalb eines Waldes in die gleiche Klassifikation eingebaut werden wie die betreffenden Waldgesellschaften selbst. Ein weiterer Fehler besteht darin, daß regionale und lokale, natürliche und naturnahe Lebensgemeinschaften mit den Artenkombinationen der Oekumene gleich behandelt werden. Diese sind ja nicht alt und konsolidiert wie die Naturvegetation und gehören zu einer ganz anders aufgebauten Einheit. Warum können wir in der Landschaftskunde keine Klassen bilden? Weil auch hier die völlig einheitlichen Objekte selten sind, weil die Einheiten gleitend ineinander übergehen, weil die von sehr vielen Faktoren abhängige Vereinigung von Komponenten infolge ihrer Einzigartigkeit und Kompliziertheit keinen Klassenwert haben kann, und weil ein einheitliches Klassifikationsprinzip fehlt. Auch hier haben die verwendeten Klassifikationsprinzipien nur lokale Wirksamkeit. Diese müssen ständig wechseln wie etwa auf der Niederrheinkarte von Paffen, wo einmal die Vegetation mit einbezogen wird, an anderer Stelle Geologie und Geomorphologie genügen muß, so daß man sich fragt, ob nicht eine gute topographische Karte mehr und objektiveres gibt. Daß die Wirksamkeit der Prinzipien nur 
partieller Art ist, gilt für die Landschaftskunde noch viel mehr als für die Vegetationskunde, weil nie die ganze Einheit von einem Divisionsgrund her erfaßt wird.

Was tritt nun an die Stelle der Klassifikation? In der Biocoenologie, in der es sich nicht um klassifikable Objekte handelt, müssen wir Typen bilden, und zwar für die Großgliederung die biogeographischen, den Breitenzonen folgenden Einheiten, die Vegetationsgürtel, und für die Kleingliederung die oekologisch-physiognomischen Formen und die Struktur der Lebensgemeinschaften. In der Landschaftskunde müssen zunächst die Naturlandschaften von den Kulturlandschaften, der Oekumene, getrennt werden. Die ersteren werden bearbeitet indem frei für irgendwelche $Z$ wecke größere oder kleinere Ausschnitte aus der Erdoberfläche ausgewählt, oder aber für Landschaftsmonographien vollständige Analysen durchgeführt werden. Für die Oekumene muß der Grad der Beeinflussung durch den Menschen nach oekonomisch-soziologischen Phasen festgelegt und dementsprechend in die vom Menschen gebildete Ganzheit eingebaut werden.

Wir dürfen Ganzheit, Einzigartigkeit, Einheitlichkeit, organismisches Gefüge und soziale Organisation nicht miteinander verwechseln. Einzigartigkeit, Einheitlichkeit bedeuten noch nicht Ganzheit, und Ganzheit noch nicht organismisches Gefüge und Organisation. Die Biocoenosen und die Landschaften sind meist Einheitlichkeiten ohne Ganzheitscharakter und können als nicht individuierbare Objekte nicht klassifiziert werden. Die Lebensgemeinschaften werden biogeographisch und oekologisch-physiognomisch und strukturell analysiert und typisiert mit biogeographischen Einheiten und mit der oekologisch-physiognomischen Form als Bezugsgrößen. Die Landschaften werden analysiert nach ihren zahlreichen Komponenten, geophysischen, geologischen, geomorphologischen, klimatologischen usw., und ihre Synthese erfolgt unter Zuhilfenahme der vollständigen Komponenten und der ganzen Erde als Bezugsgrößen, oder aber, bei monographischer Bearbeitung, mit Berücksichtigung aller dieser Charaktere. Die Oekumene, der vom Menschen veränderte Teil der Erdoberfläche, erfordert eine ganz andere begriffliche Erfassung als Biocoenose und Naturlandschaft. Sie ist im Zusammenhang mit dem Menschen zu behandeln, mit den Erkenntnissen der Anthropologie, der Ethnographie, der Soziologie, wobei dem kulturlandschaftlich interessierten Geographen die Aufgabe sich stellt, die Naturgrundlagen zu erforschen und mit den oekonomisch-soziologischen und historischen Gegebenheiten zu verknüpfen.

Bei aller Anerkennung des auf dem Gebiete der Vegetations- und Landschaftskunde von den Vertretern der klassifikatorischen Methode geleisteten Arbeiten müssen wir doch bemerken, daß das dogmatische Spielen mit fiktiven Begriffen, mit Konventionen, willkürlichen und subjektiven Abstraktionen uns nicht weiter bringt. In der Vegetationskunde wird der Ausblick auf die Großgliederung und die wesentliche oekologisch-strukturelle Kleingliederung verbaut und eine vergleichende Statistik unmöglich gemacht; wir bleiben im Lokalfloristischen stecken. In der Landschaftskunde vernachlässigen wir die Korrelation der Komponenten zum Ganzen der Erdoberfläche. Dinge wie Assoziationsindividuum, Assoziationsklasse, Ordnung gibt es in der Natur nicht, ebensowenig wie die Klassen der Paffenschen Hierarchie. Wirklich sind nur die ständig beobachtbaren Vegetationsausschnitte, die Verbreitung ihrer Teilnehmer, die vergleichend statistisch erfaßbare Partizipation an Milieu und Lebensgemeinschaften, ihre Physiognomie und ihr Zusammentreten zu Strukturen, in der Landschaftskunde die Komponenten der Ausschnitte aus der Erdoberfläche. Sie genügen für die Erfassung und Bearbeitung der Untersuchungsobjekte dieser beiden Wissenschaften.

Das Procedere soll am Beispiel des westlichen Spanischen Rifs gezeigt werden. Für die Biocoenologie beginnt die Untersuchung mit den Quadrataufnahmen der Artengarnitur. Auf Grund der approximativen Zählungen erhalten wir ein Normalquadrat mit Hilfe dessen wir die Veränderungen in den Arten- und Wuchsformen der untersuchten einheitlichen Vegetationen feststellen können. Die Artenliste wird bearbeitet durch die floristischen Analysen: die taxonomische Analyse belehrt über die ta- 
xonomische Wertigkeit und Variabilität, die phylogenetische über die Abstammung, die genetische (soweit sie vorliegt) über den Charakter der jungen Endemismen, die besonders wichtige chorologische über die Verbreitung in horizontaler und vertikaler Richtung und die epiontologische über die Geschichte der Verbreitung. Als Ergebnis dieser Analysen werden wir über die floristische Struktur des von uns gewählten Ausschnittes orientiert, über die komplizierte Zusammensetzung aus ganz heterogenen Typen von Arten der Küstengebiete auf oligotrophen Böden wie Ericoideen, Genisteen, Drosophyllum, Corema; alte isolierte Geschlechter, zum Teil in sekundärer Entwicklung begriffen, viele Endemen, einem chorologischen Typus zugehörend, welcher dem atlantischen Küstengebiet, soweit es subtropisch und feucht ist, folgt, über die Typen der Schluchten, ebenfalls mit alten Geschlechtern, welche sich in verwandten Arten verfolgen lassen über die feuchten Subtropen der nördlichen Hemisphäre hinweg mit Arten wie Prunus Laurocerasus, Hedera canariensis, Smilax mauretanica, über die Typen der sommertrockenen Mediterranwälder mit Quercus Ilex, Pinus halepensis, Pistacia-, Phillyrea-Arten. So folgen die Floren einander, abwechselnd mit den Höhenlagen, mit den Feuchtigkeits-, mit den Bodenverhältnissen bis in die Gipfelregion, wo wir nordische Arten in großer Zahl vorfinden. Die Wertigkeit der Endemismen gibt uns Auskunft über das Alter der Siedlungen, zeigt uns z. B., daß die nordischen Einwanderer größtenteils erst mit den Eiszeiten gekommen sind und zwar sowohl über die Tunis- wie auch über die Gibraltarbrücke. Arten des Fagus-Abies-Gürtels der letzten Eiszeiten zeigen keine oder nur sehr geringe Abweichungen vom Typus, so etwa Aposeris foetida oder Scilla hispanica oder Geum silvaticum, während etwa die Arten des Quercus Ilex-Gürtels und besonders die Relikte aus dem südhemisphärischen Trockengebiet (die mediterranen Stapelia-, Caralluma-Pelargonium- u. a. Arten gehören hierher) auf ein mittel- und alttertiäres Alter hinweisen. Nach den floristischen Analysen folgen die ökologisch-physiognomischen, welche die Wuchsform der Arten und ihre Zuteilung zu Typen untersuchen. Auch hier wird sofort die große Verschiedenheit zwischen den Vegetationsausschnitten sichtbar; das gleiche gilt für das abiotische Milieu und, wie uns die Strukturbeschreibung erweist, für den räumlichen Aufbau der Lebensgemeinschaften. Auf diese Weise kann jeder Vegetationsausschnitt, ob einheitlich oder nicht, analysiert werden und findet den Anschluß an die verwandten Bestände, im Falle der großen Standard-Gürtel bis über die ganze nördliche Hemisphäre hinweg. Bei der klassifikatorischen Methode endet der Gliederungsversuch im lokaleren Florengebiet und verzichtet auf die Verwendung des heute schon recht umfangreichen Analysenmateriales, so daß der Anschluß an die weltweiten Verhältnisse nicht hergestellt werden kann.

In der landschaftskundlichen Bearbeitung geht aus der Analyse der Landschaftskomponenten die gleiche Vielfältigkeit hervor. Kristallines wechselt mit Kalkgebirge und Flysch, stotzige Felsen mit weichen Formen, Podsolböden mit eutrophen, steilwandige Tobel mit breiten Mulden und Terrassen.

Aus der Vegetation können wir bereits erkennen, wie mannigfaltig auch das Klima ist. Das maritime, in der Meeresnähe völlig frostfreie Klima des Genisteen-EricoideenGürtels, das Klima der Schluchten mit den Fragmenten des Laurocerasus-Gürtels, der sommertrockene mediterrane und submediterrane Typus mit dem Quercus Ilex und dem Quercus pubescens-Gürtel, das Gebirgsklima des über 2000 m hohen Kalkstockes mit der borealen Flora, den üppigen Abieswäldern, die trockenen südexponierten Kalkfelshänge mit den Fragmenten des Quercus Ilex-Waldes. Es sei hier an die ganz ausnehmend differenten Klimaverhältnisse der Kanaren und Madeiras erinnert, welche von ebenso differenten Vegetationen begleitet werden (vergl. LAUTENSACH). Dazu kommt noch das Vordringen der Oekumene in diesen Ausschnitt, der bescheidenen Berbersiedlungen mit ihrer Selbstversorgerwirtschaft, der vorwiegend Getreide erzeugenden, merkantile Wirtschaft betreibenden Bevölkerung der höheren Bergterrassen und der stark veränderten Kulturlandschaft in der Nähe der Städte. Alle Komponenten 
stehen in einer unlöslichen Verbindung zu näheren und ferneren Fortsetzungen, die stratigraphischen wie die tektonischen, geomorphologischen, klimatologischen, edaphischen und vegetationskundlichen. Nur deren Berücksichtigung führt uns zum Verständnis unseres Ausschnittes, nur eine separate Darstellung der einzelnen Komponenten ist möglich, nicht aber die Häufung der Charaktere in einer als Ganzheit vorgeführten «normierten» Landschaft.

Anmerkingen (in Form zweier Briefe). 26. 8. 55. Lieber Herr Kollege ScHmid, herzlichen Dank dafür, daß Sie so bereitwillig und rasch mit Ihrem Aufsatz auf meine Bitte um Stellungnahme zür Diskussion über den Ganzheitsbegriff in Biologie und Landschaftskunde eingetreten sind. Ich glaube, daß er wesentlich zur Klärung beiträgt, obwohl Ihr Verzicht auf Berücksichtigung der ziemlich umfangreichen Literatur zum Thema die rasche Verständigung vielleicht verzögern mag. In einigen Punkten scheinen mir Ihre Ausführungen freilich noch Fragen offen zu lassen, deren Erörterung schon an dieser Stelle angezeigt sein dürfte. Ich wäre Ihnen deshalb dankbar, wenn Sie hierauf kurz eingingen. Zunächst wäre abzuklären, ob Ihr Ganzheitsbegriff generelle Gültigkeit beanspruchen kann. Die Divergenzen um diesen Begriff lassen dies doch etwas diskutabel erscheinen. Ich persönlich frage mich vor allem, ob er hinreicht, die prinzipielle Unterscheidung von Organismus und Landschaft völlig eindeutig zu begründen. Ich erlaube mir deshalb, dies als "erstes Echo" durch einige Hinweise auf wenige Ganzheitskriterien anzudeuten. Was 1. das Kriterium der Autonomie betrifft, sehe ich nicht ganz klar, wie Sie diesen Terminus verstanden wissen möchten. So wie Sie ihn offenbar auffassen (nämlich als ageringere» Umweltabhängigkeit des Organismus), scheint er mir zu relativ, um als Unterscheidungskriterium von Organismus und Landschaft (von den Biozönosen sehe ich hier aus Einfachheitsgründen ab) dienen zu können. Denn der Organismus (das organische Individuum) ist doch (hinsichtlich Raumbeanspruchung bzw. -verdrängung, Gravitation, Substanz- bzw. Energieaufnahme und -abgabe) so entscheidend auf das anorganische und organische, terrestrische und kosmische Milieu (Sonne) angewiesen, daß sogar das Selbstregulations- und reproduktionsvermögen (als wichtige Partialkriterien der Autonomie) so sehr eingeschränkt wird, daß nicht einmal einzusehen ist, inwiefern in diesem Rahmen überhaupt nur noch von Wahlfreiheit des Organismus gesprochen werden darf! Was geschähe mit einem Organismus, wenn man ihn in einen völlig leeren Raum brächte? Dann würde nicht nur seine Autonomie, sondern seine Existenz schlechthin völlig in Frage gestellt, m. a.W. = Null. Andererseits kann man doch auch einer Landschaft (selbst wenn sie lediglich als summative Kombination von anorganischen und organischen Erscheinungen aufgefaßt wird) eine gewisse Autonomie kaum absprechen, wenn man sich nur der allerdings andern Größenordnung und Kombination der Komponenten (im Vergleich zum Organismus) bewußt ist. In einer Kulturlandschaft z. B. reguliert eben der Mensch - der in dieser "Kombination" eine Komponente darstellt - mehr oder weniger die übrigen Komponenten; in einer Naturlandschaft vermögen je nachdem organismische oder anorganismische Teile das landschaftliche Gesamtgefüge zu "regulieren", wodurch eine gewisse Autonomie der Kombination (z.B. gegenüber der Gesamtumwelt wie gegenüber andern, benachbarten Landschaften) resultiert. - 2. Hinsichtlich des Kriteriums des abegrenzten * Gefïges (Abgrenzbarkeit, Isolierbarkeit) scheint mir dessen relative Gültigkeit bei den Organismen nicht minder evident. Zwar läßt sich offenbar grob statisch-morphologisch der Organismus durch Epidermalorgane oder -gewebe relativ leicht von seiner Umgebung abgrenzen. Wie schwer dies aber tatsächlich fällt, haben u. a. L. v. BertalanfFy (Theoretische Biologie I, 1932, 268-86) und H. Weber (Grenzen in der Biologie, Studium Generale 5, 1952, 356-363) dargetan. Vollends schwierig, wenn nicht unmöglich aber wird die «funktionelle» (physiologisch-ökologische) Isolierung, da die vorhin erwähnte Relativität der Organismusautonomie das einzelne Lebewesen geradezu absolut zum "Teil» der ganzen "Welt», die Frage wie die "Funktionseinheit" des Lebewesens zu begrenzen sei, beinahe unlösbar macht. Andererseits greifen Landschaften a) weder "unbegrenzt» in die Gashülle (Atmosphäre) noch in die Gesteinshülle (Lithosphäre) der Erde ein, (hören vielmehr, in radialer Richtung zum gesamten Erdkörper gesehen, durchaus bestimmt, wenn auch wohl kaum mathematisch-scharf fixierbar, dort auf, wo eine ihrer Hauptkomponenten [Litho-, Hydro-, Atmo- oder Biosphäre] aussetzt). Ferner differenzieren sie sich b) horizontal, indem die Kombination ihrer "Komponenten" in dieser Richtung ändert (wobei sowohl "gleitende» Übergänge: Wüste-Halbwüste-Wüstensteppe-Steppe usw. als auch "scharfe" Diskontinuitäten, z. B. Weltstadt-Urwald in der Neuen Welt usw. auftreten können). - Bei dieser Gelegenheit möchte ich anmerken, daß mir Ihr Hinweis darauf, daß Landschaften «lediglich» vom Menschen gesehene, also beliebige, willkürliche Ausschnitte aus der Erdhülle darstellten, der sichern Begründbarkeit vorderhand zu entbehren scheint; abgesehen davon, daß Sie wohl kaum überzeugend nachweisen könnten, daß ein Organismus weniger nur vom Menschen "gesehener Ausschnitt" repräsentiert, hat die bisherige Wahrnehmungspsychologie - bzw. -physiologie über die Willkürlichkeit bzw. Objektivität der Wahrnehmung von Teil-Einheiten der Gesamtwirklichkeit (und damit übrigens auch zur Frage, was Ganzheiten sind) noch nicht das letzte Wort gesprochen. - Was 3. das Kriterium der Unteilbarkeit (bzw. Teilbarkeit) anbetrifft, scheint es mir hinsichtlich des Organismus (bzw. von Ganzheiten) besonders relativen Wert beanspruchen zu dürfen. Vermag doch gerade der höchst organisierte Organismus (Mensch) weitgehende Ampu- 
tationen sehr wesentlicher Teile (Gewebe, Organe) auszuhalten, ohne daß er seiner Existenz, ja nicht einmal seines spezifischen Charakters verlustig geht. Andererseits kann eine Landschaft keineswegs ad inf. wesentlicher Komponenten beraubt werden (Problem der Verstädterung, Wüstenbildung durch anthropogene Einwirkungen: Soil Erosion usw.), ohne die Spezifität des Gefüges (der Kombination ihrer Komponenten) zu verlieren. - Mit dieser Frage scheint mir eng zusamenzuhängen die der Verknüpfungsweise (bzw. -intensität) der Teile in einem Gefüge, wobei auch das Problem der Summenhaftigkeit eine Rolle spielt. Beim Organismus scheinen die Teile (Zellen, Gewebe, Organe) so eng verknüpft zu sein, daß sie, um mit dem Physiker DE Broglie zu sprechen, jegliche Selbständigkeit aufgegeben haben und sogar zu morphologisch nur im Ganzen des Organismus denkbaren (existierbaren) hierarchischen Gefügen umgeformt sind. Bei der Landschaft hingegen ist, wenn ich Sie recht verstehe, die Verknüpfungsintensität der Komponenten so gering («adaptiv»), daß man hier gar nicht von Teilen, Komponenten sprechen kann, daß hier eben lediglich ein $\propto$ Haufe ${ }^{\nu}$ verschiedener Gebilde (eben eine bloße Summe) vorliegt. Mit andern Worten: im Organismus resultiert aus der Verbindung der Teil-Gebilde eine a höhere Einheit (Ganzheit), in der Landschaft nicht. Die Landschaft erscheint also lediglich als eine «Vielheit» von Dingen, der Organismus dagegen wäre eine Einheit von Dingen. (In gewissem Sinne weist ja schon das Wort Landschaft, bzw. der Suffix «schaft» darauf hin, insofern letzterer «Gruppe» bedeutet, woraus die «paradoxe» Situation resultiert, daß im Worte Landschaft mittelst eines Gruppierungssuffix ein Singularphänomen erzeugt ist.) Demgegenüber ist aber wiederum festzuhalten, daß weder im Organismus die Teile völlig unselbständig (Möglichkeit der Amputation!), noch in der Landschaft die «Komponenten voll selbständig sind (Änderungen der Komponenten bei «Verpflanzung», eben mittels Adaptation). - Die zweifellos zu knappen Hinweise auf einige wenige Kriterien scheinen sich $\mathrm{m}$. E. nun doch auf einen gemeinsamen Nenner bringen zu lassen, auf die Frage: Wo liegt die Grenze (der Schwellenwert), von welcher aus in der einen Richtung (nach der organismischen hin) von aganzheitlicher» Kombination der Dinge, d. h. von relativ autonomen einheitlichen Komplexen (oder «Komplexeinheiten"), nach der andern Richtung (Landschaft) nur noch von Komplexen (Mannigfaltigkeiten, Summen) gesprochen werden darf. Und da scheint mir nun allerdings das letzte Wort nicht gesagt worden zu sein. Ein solches dünkt mir vielmehr überhaupt nur dann möglich zu werden, wenn vorher die Grundbegriffe (Autonomie, Begrenztheit, Gefüge, Dauerhaftigkeit, Ganzes, Ganzheit usw.) unmißverständlich und von jedem Forscher akzeptierbar definiert worden sind. Dies ist z. Z. nicht der Fall; alle Diskussionen für oder wider Ganzheitlichkeit bzw. Summenhaftigkeit usw. müssen daher "notwendig» aneinander vorbeizielen. - Ich hoffe gerne, daß Sie meine Bedenken zerstreuen werden und bleibe inzwischen mit aufrichtigen Wünschen für erholungsreiche Ferien - die ja für Sie stets mit botanischen und landschaftlichen Entdeckungen identisch sind - im schönen Salerno Jhr E. WINKLER.

2. 9. 55. Lieber Herr Kollege, Ich habe heute die Korrekturbogen erhalten und schicke sie umgehend ... an Sie, zusammen mit den Versuchen, Ihre Einwendungen zu entkräften. - Als autonom wird allgemeinst ein ganzheitliches Objekt bezeichnet, welches auf äußere Einwirkungen nicht direkt reagiert, sondern über ein System von Potenzen, Reserven, Relais, ein Objekt, welches so gepuffert ist, daß es ein \pm umfangreiches Eigenleben besitzt. Nur die lebenden Komponenten einer Landschaft oder die Kulturlandschaft reagieren so. Für die letztere gilt das nur, wenn es sich nicht um Raubbaugebiete handelt. Für die ersteren ist zu sagen, daß eine mächtige Vegetationsdecke allenfalls noch einen retardierenden Einfluß ausüben kann; das ist aber auch alles. Es handelt sich nicht um Größenordnungen, sondern um Qualitäten und um Niveaus. - Die Kulturlandschaften sind begrifflich völlig von den Naturlandschaften zu trennen. Der Mensch ist mehr als eine Komponente, ein das Ganze organisierendes Element, so wie im Kleinen das Tier im Termitenbau oder im Biberbau. Er wandelt die nicht ganzheitliche Naturlandschaft in die ganzheitliche Ökumene um. Mit dem Einfluß des Menschen stellt sich auch eine Umgrenzung in der Kulturlandschaft ein, welche wir in der Biocoenologie ohne weiteres konstatieren können. - Die Begrenzung der Naturlandschaften in der Vertikalen gilt für alle Ausschnitte gleich und fällt außer Frage. In der Horizontalen gibt es keine wesentlichen, d. h. aus dem Charakter der Einheit entstehende Grenzen. Sie laufen immer via Komponenten. Das Beispiel Weltstadt-Urwald hat damit nichts zu tun, denn hier grenzt die Ganzheit der Ökumene an die Nichtganzheit Naturlandschaft. Das gilt auch für das, was Sie von der Verstädterung und von der „Badland“-Bildung sagen. - Die Betrachtungen über die Begrenzung des Organismus sind mehr eine Angelegenheit der Physiologie und theoretischen Ökologie und berühren uns in diesem Zusammenhange kaum. Für unsere Praxis genügt es vollkommen, daß wir jederzeit einen pflanzlichen oder tierischen Organismus und sein Territorium wahrnehmen können, ebenso die Landschaftskomponenten und die Ökumene des Menschen. Was schwierig ist, das sind die Rekonstruktionen der Natur bei Biocoenose und Landschaft in der Vergangenheit. Beachten Sie, daß in der botanischen und zoologischen Taxonomie die Grundbegriffe Species, Gattung usw. immer noch heftig diskutiert werden und trotzdem eine ersprießliche systematische Arbeit geleistet wird. Wenn wir darauf warten müßten, bis die Begriffe in Biocoenologie und Landschaftskunde bereinigt wären, kämen wir überhaupt nicht zu unserer Arbeit. Für unsere Zwecke genügt das, was an Divisionsgründen vorliegt, auch für eine, seriöse wissenschaftlichen Ansprüchen standhaltende Bearbeitung. Halten wir uns an die objektiv erkennbaren, immer wieder kontrollierbaren Dinge, ganz in dem Sinne, wie Sie sagten, daß es die jungen Geographen machen.. Mit den besten Grüßen bin ich Ihr E. Schмid. 
Dieser Entkräftungsversuch zeigt, da $ß$ offenbar nach wie vor unbeantwortete Fragen bleiben. Eines scheint mir immerhin aus ihm klar hervorzugehen, daß nämlich das Problem «Ganzheitlichkeit des Organismus - Summenhaftigkeit der Landschaft [und zwar der Natur- und Kulturlandschaft]» nicht ohne weiteres zugunsten des einen bzw. zu Ungunsten des andern Phänomens zu erledigen ist. Mich persönlich dünkt, die Lösung dürfte in einem mittleren Bereiche zu suchen und zu finden sein. M. a.W. die Landschaften sind für mich ebenso Vielheiten und Einheiten wie Organismen (und alle konkreten Gebilde der Wirklichkeit), wobei klar sein dürfte, daß zwischen beiden unzweifelhaft essentielle Unterschiede bestehen - ansonst sie der Mensch ja auch gar nicht verschieden benannt hätte. Ob hierbei Organismen mehr Einheiten als Vielheiten, Landschaften mehr Vielheiten denn Einheiten darstellen, scheint mir - ich vermute damit auch im Sinne E. Schmids zu sprechen - in diesem Zusammenhang nicht entscheidend wichtig (wobei ja, völlig konkret gesehen, jeder Organismus ein solch vielfältiges Forschungsobjekt ist, daß die Einheit vorderhand faktisch nicht weniger ein Problem bleibt als bei der Landschaft!). Andererseits ist die Frage der Einheit der Landschaft immerhin weder theoretisch noch praktisch, weder für ihre Erkenntnis noch für ihre Planung, Gestaltung und Nutzung belanglos. Es ist keineswegs gleichgültig, ob Landschaft a) nur als Einheit gelten kann (bei der die Teile völlig unselbständig sind und daher überhaupt vernachlässigt werden können), ob sie b) nur Summe darstellt (bei der keinerlei Bindungen der Komponenten aneinander und zum "Ganzen" zu berücksichtigen sind), oder ob sie c) Einheit und Vielheit zugleich (mannigfaltige Einheit oder einheitliche Mannigfaltigkeit) ist, bei welcher «Struktur» Teile und Ganzes ihrer Bedeutung gemäß zu würdigen sind, wenn realiter eine sinnvolle, «optimale» Existenz beider erzielt werden soll. Ich erblicke gerade in der Tatsache, daß Prof. SCHMID sich von jeher nicht allein, ja nicht einmal vorzugsweise für den Schutz einzelner Organismen, sondern für denjenigen "ganzer» Biozönosen (und damit im Grund für Landschaften) eingesetzt hat und dauernd einsetzt, einen sehr konkreten und wertvollen Beweis dafür, daß $\mathrm{ihm}$ offenbar der Zusammenhang aller Dinge - worum es eigentlich bei der Kontroverse Ganzheit - (die m. E. an realen Dingen immer nur relativ erfüllt ist: welcher Organismus ist voll ganzheitlich ?) Summenhaftigkeit geht, - ihr Gefüge, doch nicht weniger real und wichtig erscheint als die «Einzelgebilde " selbst, und glaube, daß in dieser Hinsicht die Differenzen zwischen den Forschern im Grunde mehr formalen als materialen Charakter tragen. E. W.

\section{LA CONCEPTION D'INTEGRALITE DANS LA BIOCENOLOGIE ET DANS LA SCIENCE DU PAYSAGE}

La conception d'intégralité est discutée ici en relation avec la biocénologie et la science du paysage. L'application de cette conception à la biocénose et au paysage est inadéquate. Il est impossible de créer des classes et des systèmes de classification. Les phénomènes biocénologiques et géographiques, quelle que soit leur nature, même s'ils sont homogènes ou uniques, ne peuvent pas être considérés comme indivisibles ou comme un organisme. L'analyse des biocénoses et des paysages doit donc être effectuée par celle des participants et des composants. Les biocénoses et les paysages ne sont que des tranches plus ou moins homogènes, l'une de la végétation, l'autre de la superficie de la terre. Le paysage déformé par l'influence de l'homme doit être traité séparément du paysage naturel, lorsque les déformations sont prédominantes.

\section{DIE LANDSCHAFT IM UNTERRICHT DER MITTELSCHULE*}

\section{Werner Nigg}

Über «Landschaft》 $z u$ sprechen, erscheint im Rahmen eines Kurzvortrages beinahe unmöglich. Nicht zuletzt deshalb, weil der Begriff heute noch immer Gegenstand lebhafter Auseinandersetzungen bildet. Obwohl es m. E. falsch wäre, diese wissenschaftlichen Kontroversen in die Mittelschule zu tragen, da die Geographie an der Mittelschule ihrem Wesen und Ziel nach doch etwas anderes darstellt als an der

* Dieser Aufsatz entspricht dem Vortrag «Die Behandlung einer Landschaft in der Mittelschule», der am 15. Januar 1955 an der Arbeitstagung des VSGg in Olten gehalten wurde (vgl. GH 1955, p. 34). Er war ausschließlich als Anregung zur Diskussion über ein Thema gedacht, das gewiß nicht nur zu den zentralsten des Geographieunterrichtes, sondern der Geographie überhaupt gehört. Es läge wohl im Interesse der Mittelschulgeographie, wenn die Publikation dieses Kurzvortrages zur Weiterführung der Diskussion - die an der Tagung nicht über einige Voten hinauskam - veranlassen würde. 